\title{
Quale accesso vascolare per il futuro?
}

\author{
Luciano Carbonari
}

\author{
Clinica di Chirurgia Vascolare, A.O. Umberto I, Ancona
}

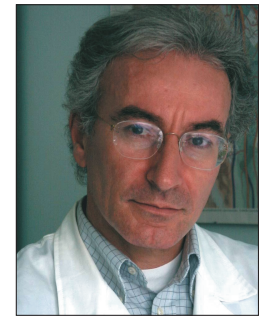

$\mathbf{L}$ qualità della vita del paziente emodializzato si identifica sempre di più con la "bontà" dell' accesso vascolare. Un accesso vascolare precario, o mal funzionato, è fonte di ansia per il paziente, e di preoccupazione per il personale sanitario preposto alla emodialisi; è inoltre fonte di complicanze e patologie associate.

Oggi è unanimemente accettato che la fistola artero-venosa con vasi nativi rappresenti l'accesso emodialitico d'elezione.

Negli ultimi anni in realtà il problema dell'accesso vascolare si è riproposto prepotentemente in quanto la vita dialitica dei pazienti si è prolungata in modo considerevole e il patrimonio venoso idoneo per l'accesso vascolare in molti casi si è esaurito.

Anche se la tecnologia negli ultimi anni ha prodotto protesi e cateteri innovativi, questi rappresentano soltanto un ripiego quando i vasi del paziente non sono più reperibili. I facili entusiasmi nei confronti di ogni nuo- vo presidio (protesi, cateteri o sistemi totalmente impiantabili), spesso caldeggiati da aziende fornitrici e a volte condivisi troppo precipitosamente da qualche sperimentatore, non devono far dimenticare che l'organismo tende a rifiutare tutto ciò che è estraneo, inoltre negli anni ci si è dovuti spesso confrontare con la loro scarsa durata e soprattutto con una nuova serie di patologie indotte.

Dunque non vi sono dubbi su quale dovrà essere l'accesso vascolare del futuro.

Il problema che si pone è di come utilizzare al meglio il patrimonio vascolare del paziente.

A questo proposito mi permetto di suggerire alcune regole comportamentali:

1) Sviluppare la cultura del rispetto del patrimonio venoso.

I pazienti arrivano spesso all'emodialisi dopo terapie infusive prolungate o interventi chirurgici. Il rispetto del patrimonio venoso presuppone la conoscenza della lesività delle soluzioni infuse e dei presidi utilizzati. La scelta e l'uso della vena dovrebbero essere guidati dalla logica del massimo rispetto piuttosto che da quella del "più facile da aggredire". Il raggiungimento di questo scopo richiede il coinvolgimento di tutto il personale sanitario sia medico che infermieristico a partire dalla scuola di formazione.

2) Corretta progettazione dell'accesso vascolare in rapporto alle aspettative di vita e al patrimonio vascolare disponibile.

Quando per il paziente si prefigura la necessità di un trattamento dialitico, è compito fondamentale del nefrologo, analizzare le aspettative del paziente considerando ogni possibile variabile: età, patrimonio vascolare disponibile (arterioso e venoso), malattie associate, anni di dialisi previsti, possibilità del trapianto ecc.

La progettazione è senza dubbio il compito più difficile di tutte le problematiche inerenti all'acceso vascolare in quanto non risponde a regole fisse, ma scaturisce dall'esperienza individuale. È importante saper prevedere l'evoluzione dei vasi utilizzati, conoscere le capacità gestionali dell'accesso nel Centro di dialisi, in definitiva saper prevedere la durata della fistola, avere una chiara soluzione per ogni possibile complicanza che con- 
senta di poter prevedere evoluzione e durata della fistola.

3) Corretta esecuzione dell'acceso vascolare:

la cultura nefrologica è prevalentemente medica e non è prevista un'attitudine manuale come nelle scuole chirurgiche.

D'altra parte l'esecuzione solo saltuaria di interventi non consente l'affinamento della tecnica.

Tutto questo ha portato all'affidamento delle procedure ai chirurghi, soprattutto vascolari, magari con la delega anche della scelta del tipo di accesso vascolare.

Il chirurgo raramente conosce le problematiche emodialitiche, spesso esegue delle anastomosi artero-venose, magari in modo ottimale, ma senza immaginare la possibilità di utilizzo e soprattutto senza conoscere il "progetto intorno al paziente".

E tempo che il nefrologo si riappropri del ruolo di Responsabile dell'accesso vascolare, che collabori con le diverse discipline per realizzare al meglio quanto previsto dal "suo progetto".

4) Corretta gestione, monitoraggio e manutenzione dell'accesso vascolare.

L'accesso vascolare alla pari delle macchine per emodialisi deve essere considerato uno strumento e come tutti gli strumenti, si usura durante l'utilizzo, si danneggia se non usato correttamente; deve essere quindi sottoposto a controllo e riparato se mal funzionante.

Tutto questo richiede conoscenza dei problemi, attenzione, impegno.

Purtroppo la precarietà del personale sanitario con un sempre più rapido tournover ostacola la continuità di un rapporto con il paziente, presupposto fondamentale per un buon monitoraggio. Anche in questo settore il ruolo del nefrologo è fondamentale, deve saper percepire precocemente i mal funzionamenti, avere la cultura delle possibili soluzioni avvalendosi di specialisti radiologi e chirurghi per la correzione.

Quanto esposto può sembrare un elenco di banalità; tutto sembra scontato. In realtà, di fronte a un paziente con problema di accesso vascolare, ci si accorge che quasi sempre molte di queste raccomandazioni sono state disattese.

Dobbiamo tutti convincerci che le innovazioni tecnologiche, seppure auspicabili, non potranno incidere in modo significativo sul futuro dell'accesso vascolare.

Le protesi non sono adatte per essere punte, hanno una durata molto limitata e sono gravate da complicanze.

I cateteri, per quanto migliorabili, non potranno mai rappresentare un'alternativa.

La fistola con vasi nativi rappresenta e rappresenterà il gold standard dell'accesso vascolare. Il problema attuale e futuro non è quindi quello di trovare una soluzione migliore, è invece quello di garantire questa soluzione per tutta la durata della vita dialitica.

Soltanto se ci convinceremo di questo potremo capire fino in fondo l'importanza del rispetto delle regole di comportamento suggerite.

Il mio convincimento è che se ben utilizzato il patrimonio venoso individuale è, e sarà, in grado di garantire un accesso vascolare idoneo nella grande maggioranza dei pazienti.

La realizzazione di quanto esposto richiede un grande sforzo collettivo.

Ancora oggi l'accesso vascolare viene eseguito e gestito in modo "artigianale" utilizzando esperienze e professionalità locali, realizzando ciò che si è in grado di fare piuttosto che ciò che si dovrebbe fare. Soltanto un ampio dibattito potrà far emergere linee di comportamento comunemente accettate. A questa prima fase che dovrà coinvolgere tutti i nefrologi dovrà seguirne una seconda, di tipo operativo coinvolgente altre branche specialistiche: chirurghi, radiologi, anestesisti ecc.

Si dovrà stabilire come e chi realizzerà gli accessi vascolari sia quelli semplici che quelli complessi, magari creando una rete territoriale di riferimento.

È compito delle comunità scientifiche fare in modo che il paziente emodializzato abbia lo stesso trattamento a prescindere dal Centro a cui afferisce. Il progetto è ambizioso ma proprio per questo molto stimolante.

carboelle@yahoo.com 